\title{
ASYMPTOTIC BEHAVIOR OF SOLUTIONS FOR NEUTRAL DYNAMIC EQUATIONS ON TIME SCALES
}

\author{
DOUGLAS R. ANDERSON
}

Received 30 January 2006; Revised 17 March 2006; Accepted 17 March 2006

We investigate the boundedness and asymptotic behavior of a first-order neutral delay dynamic equation on arbitrary time scales, extending some results from difference equations.

Copyright (c) 2006 Douglas R. Anderson. This is an open access article distributed under the Creative Commons Attribution License, which permits unrestricted use, distribution, and reproduction in any medium, provided the original work is properly cited.

\section{Neutral delay dynamic equation}

We consider, on arbitrary time scales, the neutral delay dynamic equation

$$
[x(t)-p(t) x(k(t))]^{\Delta}+q(t) x(\ell(t))=0, \quad t \in\left[t_{0}, \infty\right)_{\mathbb{T}},
$$

where $\mathbb{T}$ is a time scale unbounded above, the variable delays $k, \ell:\left[t_{0}, \infty\right)_{\mathbb{T}} \rightarrow \mathbb{T}$ are nondecreasing with $k(t), \ell(t)<t$ for all $t \in\left[t_{0}, \infty\right)_{\mathbb{T}}$ such that $\lim _{t \rightarrow \infty} k(t), \ell(t)=\infty$. The coefficient functions $p, q: \mathbb{T} \rightarrow \mathbb{R}$ are right-dense continuous with $p$ bounded and $q \geq 0$. To clarify some notation, take $\ell^{-1}(t):=\sup \{s: \ell(s) \leq t\}, \ell^{-(n+1)}(t)=\ell^{-1}\left(\ell^{-n}(t)\right)$ for $t \in$ $\left[\ell\left(t_{0}\right), \infty\right)_{\mathbb{T}}$, and $\ell^{n+1}(t)=\ell\left(\ell^{n}(t)\right)$ for $t \in\left[\ell^{-3}\left(t_{0}\right), \infty\right)_{\mathbb{T}}$. For $p$ and $k$ above, let $\Omega$ be the linear set of all functions given by

$$
\Omega:=\left\{x: \mathbb{T} \rightarrow \mathbb{R}:[x(t)-p(t) x(k(t))]^{\Delta} \in C_{r d}\left(\left[t_{0}, \infty\right)_{\mathbb{T}} ; \mathbb{R}\right)\right\} ;
$$

solutions of (1.1) will belong to $\Omega$.

In the aftermath of Hilger's breakthrough paper [4], a rapidly diversifying body of literature has sought to unify, extend, and generalize ideas from discrete calculus, continuous calculus, and quantum calculus to arbitrary time-scale calculus, where a time scale is merely a nonempty closed set of real numbers. This paper illustrates this new understanding by extending some discrete results from difference equations to dynamic equations on time scales. In particular, (1.1) is studied in [6] with $\mathbb{T}=\mathbb{Z}$ and $p \equiv 0$, and in [5] in the case when $\mathbb{T}=\mathbb{Z}$ with variable $p$. Much of the organization of and motivation for this paper arise from $[5,6]$. For more on delay dynamic equations, see, for

Hindawi Publishing Corporation

Advances in Difference Equations

Volume 2006, Article ID 80850, Pages 1-11

DOI 10.1155/ADE/2006/80850 
example, [1]; for more on time scales, jump ahead to the appendix, or consult the recent texts $[2,3]$.

\section{Vanishing of solutions at infinity}

Recall that in this paper we consider only the case where the coefficient function $p$ in (1.1) is nonconstant but bounded. Before stating the main results, we need the following lemma, which is a version of the integration-by-parts formula from continuous calculus, extended to arbitrary time scales; note the interesting dependence on the graininess function $\mu$ in the last term.

Lemma 2.1 (integration by parts). For right-dense continuous functions $q: \mathbb{T} \rightarrow \mathbb{R}$ and points $a, t \in \mathbb{T}$,

$$
\int_{a}^{t}\left(q(s) \int_{a}^{\sigma(s)} q(z) \Delta z\right) \Delta s=\frac{1}{2}\left(\int_{a}^{t} q(s) \Delta s\right)^{2}+\frac{1}{2} \int_{a}^{t} \mu(s) q^{2}(s) \Delta s .
$$

Proof. Let

$$
Q(t):=\frac{1}{2}\left(\int_{a}^{t} q(s) \Delta s\right)^{2}+\frac{1}{2} \int_{a}^{t} \mu(s) q^{2}(s) \Delta s-\int_{a}^{t}\left(q(s) \int_{a}^{\sigma(s)} q(z) \Delta z\right) \Delta s .
$$

Then $Q(a)=0$, and

$$
Q^{\Delta}(t)=\frac{1}{2} q(t)\left(\int_{a}^{t} q(s) \Delta s-\int_{a}^{\sigma(t)} q(s) \Delta s+\mu(t) q(t)\right) .
$$

Since $\int_{a}^{\sigma(t)}=\int_{a}^{t}+\int_{t}^{\sigma(t)}$ and $\int_{t}^{\sigma(t)} q(s) \Delta s=\mu(t) q(t)$ using [2, Theorem 1.75], $Q^{\Delta}(t) \equiv 0$. By the uniqueness of solutions to initial value problems, $Q(t) \equiv 0$ and the conclusion follows.

For example, if $\mathbb{T}=\mathbb{R}$, then the graininess is zero and the simple formula is

$$
\int_{a}^{t} q(s)\left(\int_{a}^{s} q(z) d z\right) d s=\frac{1}{2}\left(\int_{a}^{t} q(s) d s\right)^{2} ;
$$

when $\mathbb{T}=\mathbb{Z}$, we have $\mu(t) \equiv 1$ and

$$
\sum_{k=a}^{t-1} \sum_{j=a}^{k} q_{k} q_{j}=\frac{1}{2}\left(\sum_{k=a}^{t-1} q_{k}\right)^{2}+\frac{1}{2} \sum_{k=a}^{t-1} q_{k}^{2}
$$

On a quantum time scale, $\mathbb{T}=\left\{1, r, r^{2}, r^{3}, \ldots\right\}$ for some $r>1$, so that the graininess is increasing. Interpret the points $a$ and $t$ as $r^{a}$ and $r^{t}$ for positive integers $a$ and $t$ with $t>a$. Then we have

$$
\sum_{k=a}^{t-1} \sum_{j=a}^{k} r^{k+j} q\left(r^{k}\right) q\left(r^{j}\right)=\frac{1}{2}\left(\sum_{k=a}^{t-1} r^{k} q\left(r^{k}\right)\right)^{2}+\frac{1}{2} \sum_{k=a}^{t-1}\left(r^{k} q\left(r^{k}\right)\right)^{2} .
$$


As a final example, we consider the time scale $\mathbb{T}=\left\{\sum_{n=1}^{k} 1 / n: k \in \mathbb{N}\right\}$ of harmonic numbers, where the graininess is decreasing; the result may then be viewed as

$$
\begin{aligned}
& \sum_{k=a}^{t-1} \sum_{j=a}^{k} \frac{1}{(k+1)(j+1)} q\left(\sum_{n=1}^{k} \frac{1}{n}\right) q\left(\sum_{n=1}^{j} \frac{1}{n}\right) \\
& =\frac{1}{2}\left(\sum_{k=a}^{t-1} \frac{1}{k+1} q\left(\sum_{n=1}^{k} \frac{1}{n}\right)\right)^{2}+\frac{1}{2} \sum_{k=a}^{t-1}\left(\frac{1}{k+1} q\left(\sum_{n=1}^{k} \frac{1}{n}\right)\right)^{2}
\end{aligned}
$$

for positive integers $a$ and $t$ with $t>a$.

Theorem 2.2. Suppose there exists a constant $\bar{p} \in(0,1 / 2]$ such that $|p(t)| \leq \bar{p}$ for all $t \in \mathbb{T}$, and that for large $t \in \mathbb{T}$,

$$
0<\bar{p}<\frac{1}{4}, \quad \int_{\ell(t)}^{\sigma(t)} q(s) \Delta s \leq \frac{3}{2}-2 \bar{p}
$$

or

$$
\frac{1}{2} \leq \bar{p} \leq \frac{1}{2}, \quad \int_{\ell(t)}^{\sigma(t)} q(s) \Delta s \leq \sqrt{2(1-2 \bar{p})}
$$

Then every solution $x \in \Omega$ of (1.1) is bounded.

Proof. Find $t_{1} \in \mathbb{T}$ large enough, say $t_{1}>k^{-1}\left(\ell^{-1}\left(t_{0}\right)\right)$, such that

$$
\int_{\ell(t)}^{\sigma(t)} q(s) \Delta s \leq\left\{\begin{array}{l}
A=\frac{3}{2}-2 \bar{p}: 0<\bar{p}<\frac{1}{4}, \\
B=\sqrt{2(1-2 \bar{p})}: \frac{1}{4} \leq \bar{p} \leq \frac{1}{2},
\end{array} \quad t \in\left[t_{1}, \infty\right)_{\mathbb{T}} .\right.
$$

Suppose that, contrary to the asserted conclusion, $x$ is an unbounded solution of (1.1). Set

$$
z(t):=x(t)-p(t) x(k(t)), \quad t \in\left[t_{0}, \infty\right)_{\mathrm{T}} .
$$

Then there exists $t^{*} \in\left(k^{-1}\left(\ell^{-2}\left(t_{1}\right)\right), \infty\right)_{\mathbb{J}}$ large enough such that

$$
\left|x\left(t^{*}\right)\right|>\frac{\left|z\left(\ell^{-2}\left(t_{1}\right)\right)\right|}{2(1-\bar{p})}, \quad\left|x\left(t^{*}\right)\right|>\sup \left\{|x(t)|: t \in\left[t_{0}, t^{*}\right)_{\mathbb{T}}\right\} .
$$

Then

$$
\left|z\left(t^{*}\right)\right|=\left|x\left(t^{*}\right)-p\left(t^{*}\right) x\left(k\left(t^{*}\right)\right)\right|>(1-\bar{p})\left|x\left(t^{*}\right)\right|>\frac{1}{2}\left|z\left(\ell^{-2}\left(t_{1}\right)\right)\right| .
$$

Without loss of generality, assume that $z\left(t^{*}\right)>0$. Then by (2.13), there exist points $T \in$ $\mathbb{\pi}$ and $t^{\dagger} \in[\ell(T), T)_{\mathbb{T}}$ such that

$$
z(T)=\max \left\{z(t): t \in\left[\ell^{-2}\left(t_{1}\right), t^{*}\right]_{\mathbb{T}}\right\}, \quad z^{\Delta}\left(t^{\dagger}\right)>0 .
$$


$4 \quad$ Neutral dynamic equations

Set

$$
y(t):=z(t)-\bar{p}\left|x\left(t^{*}\right)\right| \quad \text { for } t \in\left[\ell\left(t_{1}\right), \infty\right)_{\mathbb{T}} .
$$

It follows that

$$
x(\ell(t))=z(\ell(t))+p(\ell(t)) x(\ell(k(t))) \geq z(\ell(t))-\bar{p}\left|x\left(t^{*}\right)\right|=y(\ell(t)), \quad t \in\left[t_{1}, T\right]_{\mathbb{T}}
$$

(actually $\left.t \in\left[t_{1}, k^{-1}\left(\ell^{-1}\left(t^{*}\right)\right)\right]_{\mathbb{T}}\right)$ so that

$$
y^{\Delta}(t)=z^{\Delta}(t)=-q(t) x(\ell(t)) \leq-q(t) y(\ell(t)), \quad t \in\left[t_{1}, T\right]_{\mathbb{T}},
$$

using (1.1), (2.11), and (2.13). Since $\bar{p} \in(0,1 / 2]$,

$$
y(T) \geq z\left(t^{*}\right)-\bar{p}\left|x\left(t^{*}\right)\right|>(1-2 \bar{p})\left|x\left(t^{*}\right)\right| \geq 0,
$$

but by the selection of $t^{\dagger}$, by (1.1), and (2.15),

$$
0<z^{\Delta}\left(t^{\dagger}\right)=-q\left(t^{\dagger}\right) x\left(\ell\left(t^{\dagger}\right)\right) \leq-q\left(t^{\dagger}\right) y\left(\ell\left(t^{\dagger}\right)\right), \quad z^{\Delta}\left(t^{\dagger}\right)=y^{\Delta}\left(t^{\dagger}\right)
$$

Consequently $y\left(\ell\left(t^{\dagger}\right)\right)<0$ and $y(T)>0$, so that by the intermediate value theorem [2, Theorem 1.115], there exists $t_{2} \in\left[\ell\left(t^{\dagger}\right), T\right)_{\mathbb{T}}$ such that either $y\left(t_{2}\right)<0<y^{\sigma}\left(t_{2}\right)$ or $y\left(t_{2}\right)=$ 0 . Either way, $y\left(t_{2}\right) y^{\sigma}\left(t_{2}\right) \leq 0$, hence there exists a real number $\xi \in(0,1]$ such that

$$
y^{\sigma}\left(t_{2}\right)-\xi\left[y^{\sigma}\left(t_{2}\right)-y\left(t_{2}\right)\right]=y\left(t_{2}\right)+(1-\xi)\left[y^{\sigma}\left(t_{2}\right)-y\left(t_{2}\right)\right]=0 .
$$

From (2.17), we have $y^{\Delta}(s) \leq q(s)\left|x\left(t^{*}\right)\right|$ for $s \in\left[t_{1}, T\right]_{\mathbb{T}}$; integrating this from $\ell(t)$ to $t_{2}$ and using (2.20) and Theorem A.4, we obtain for $t \in\left[t_{2}, T\right)_{\mathbb{T}}$ that

$$
\begin{aligned}
-y(\ell(t)) & =\int_{\ell(t)}^{t_{2}} y^{\Delta}(s) \Delta s+(1-\xi) \mu\left(t_{2}\right) y^{\Delta}\left(t_{2}\right) \\
& \leq\left|x\left(t^{*}\right)\right|\left(\int_{\ell(t)}^{\sigma\left(t_{2}\right)} q(s) \Delta s-\xi \mu\left(t_{2}\right) q\left(t_{2}\right)\right) .
\end{aligned}
$$

Combine this with (2.17) to get

$$
y^{\Delta}(t) \leq\left|x\left(t^{*}\right)\right| q(t)\left(\int_{\ell(t)}^{\sigma\left(t_{2}\right)} q(s) \Delta s-\xi \mu\left(t_{2}\right) q\left(t_{2}\right)\right), \quad t \in\left[t_{2}, T\right)_{\mathbb{T}} .
$$

In order to contradict (2.18), we now show that $y(T) \leq(1-2 \bar{p})\left|x\left(t^{*}\right)\right|$ in the following three cases. 
Case 1. Assume that $0<\bar{p}<1 / 4$ and $\int_{\sigma\left(t_{2}\right)}^{T} q(s) \Delta s+\xi \mu\left(t_{2}\right) q\left(t_{2}\right) \leq 1$. Then

$$
\begin{aligned}
& y(T) \stackrel{\mathrm{FTC}}{=} \int_{\sigma\left(t_{2}\right)}^{T} y^{\Delta}(s) \Delta s+y^{\sigma}\left(t_{2}\right) \\
& \stackrel{(2.20)}{=} \int_{\sigma\left(t_{2}\right)}^{T} y^{\Delta}(s) \Delta s+\xi \mu\left(t_{2}\right) y^{\Delta}\left(t_{2}\right) \\
& \stackrel{(2.22)}{\leq}\left|x\left(t^{*}\right)\right|\left\{\int_{\sigma\left(t_{2}\right)}^{T} q(s)\left(\int_{\ell(s)}^{\sigma\left(t_{2}\right)} q(z) \Delta z-\xi \mu\left(t_{2}\right) q\left(t_{2}\right)\right) \Delta s\right. \\
& \left.\quad+\xi \mu\left(t_{2}\right) q\left(t_{2}\right)\left(\int_{\ell\left(t_{2}\right)}^{\sigma\left(t_{2}\right)} q(s) \Delta s-\xi \mu\left(t_{2}\right) q\left(t_{2}\right)\right)\right\} .
\end{aligned}
$$

By the property of delta integrals $\int_{a}^{b}+\int_{b}^{c}=\int_{a}^{c}$,

$$
\begin{gathered}
y(T) \leq\left|x\left(t^{*}\right)\right|\left\{\int_{\sigma\left(t_{2}\right)}^{T} q(s)\left(\int_{\ell(s)}^{\sigma(s)} q(z) \Delta z-\int_{\sigma\left(t_{2}\right)}^{\sigma(s)} q(z) \Delta z-\xi \mu\left(t_{2}\right) q\left(t_{2}\right)\right) \Delta s\right. \\
\left.+\xi \mu\left(t_{2}\right) q\left(t_{2}\right)\left(\int_{\ell\left(t_{2}\right)}^{\sigma\left(t_{2}\right)} q(s) \Delta s-\xi \mu\left(t_{2}\right) q\left(t_{2}\right)\right)\right\} .
\end{gathered}
$$

Multiplying terms, rearranging them, then using (2.10) and Lemma 2.1 yield

$$
\begin{aligned}
y(T) \leq\left|x\left(t^{*}\right)\right|\left\{A \int_{\sigma\left(t_{2}\right)}^{T} q(s) \Delta s-\frac{1}{2}\left(\int_{\sigma\left(t_{2}\right)}^{T} q(s) \Delta s\right)^{2}-\frac{1}{2} \int_{\sigma\left(t_{2}\right)}^{T} \mu(s) q^{2}(s) \Delta s\right. \\
\left.+\xi \mu\left(t_{2}\right) q\left(t_{2}\right)\left(A-\int_{\sigma\left(t_{2}\right)}^{T} q(s) \Delta s-\xi \mu\left(t_{2}\right) q\left(t_{2}\right)\right)\right\} \\
=\left|x\left(t^{*}\right)\right|\left\{A\left[\int_{\sigma\left(t_{2}\right)}^{T} q(s) \Delta s+\xi \mu\left(t_{2}\right) q\left(t_{2}\right)\right]-\frac{1}{2}\left(\int_{\sigma\left(t_{2}\right)}^{T} q(s) \Delta s+\xi \mu\left(t_{2}\right) q\left(t_{2}\right)\right)^{2}\right. \\
\left.-\frac{1}{2}\left(\int_{\sigma\left(t_{2}\right)}^{T} \mu(s) q^{2}(s) \Delta s+\left[\xi \mu\left(t_{2}\right) q\left(t_{2}\right)\right]^{2}\right)\right\} \\
\leq\left|x\left(t^{*}\right)\right|\left(A\left[\int_{\sigma\left(t_{2}\right)}^{T} q(s) \Delta s+\xi \mu\left(t_{2}\right) q\left(t_{2}\right)\right]-\frac{1}{2}\left(\int_{\sigma\left(t_{2}\right)}^{T} q(s) \Delta s+\xi \mu\left(t_{2}\right) q\left(t_{2}\right)\right)^{2}\right) .
\end{aligned}
$$

Let $w(z):=A z-(1 / 2) z^{2}$, where $z=\int_{\sigma\left(t_{2}\right)}^{T} q(s) \Delta s+\xi \mu\left(t_{2}\right) q\left(t_{2}\right) \leq 1$. Then $w^{\prime}(1)>0$ by the choice of $A$ and the fact that in this case $\bar{p}<1 / 4$. As a result,

$$
y(T) \leq\left|x\left(t^{*}\right)\right|\left(A-\frac{1}{2}\right)=\left|x\left(t^{*}\right)\right|(1-2 \bar{p}) .
$$


Case 2. Assume that $0<\bar{p}<1 / 4$ and $\int_{\sigma\left(t_{2}\right)}^{T} q(s) \Delta s+\xi \mu\left(t_{2}\right) q\left(t_{2}\right)>1$. Actually, from $\xi \leq 1$, we have in this case that $\int_{t_{2}}^{T} q(s) \Delta s>1$. Note that

$$
g(t):=\int_{t}^{T} q(s) \Delta s-1, \quad t \in\left[t_{2}, T\right]_{\mathbb{T}},
$$

is a delta-differentiable and decreasing function, so that $g$ is continuous [2, Theorem 1.16(i) ] on $t \in\left[t_{2}, T\right]_{\mathbb{T}}$. Since $g\left(t_{2}\right)>0$ and $g(T)=-1<0$, by the intermediate value theorem [2, Theorem 1.115], there exists $t_{3} \in\left[t_{2}, T\right)_{\mathbb{U}}$ such that either $g\left(t_{3}\right)=0$ or $g\left(t_{3}\right)>$ $0>g^{\sigma}\left(t_{3}\right)$. Either way,

$$
\int_{\sigma\left(t_{3}\right)}^{T} q(s) \Delta s<1 \leq \int_{t_{3}}^{T} q(s) \Delta s=\mu\left(t_{3}\right) q\left(t_{3}\right)+\int_{\sigma\left(t_{3}\right)}^{T} q(s) \Delta s,
$$

therefore there exists a real number $\eta \in(0,1]$ such that

$$
\int_{\sigma\left(t_{3}\right)}^{T} q(s) \Delta s+\eta \mu\left(t_{3}\right) q\left(t_{3}\right)=1
$$

Recall from (2.17) that

$$
y^{\Delta}(s) \leq q(s)\left|x\left(t^{*}\right)\right| \quad \text { for } s \in\left[t_{1}, T\right]_{\mathbb{T}}
$$

then

$$
\begin{aligned}
& y(T) \stackrel{(2.20)}{=} \int_{\sigma\left(t_{2}\right)}^{T} y^{\Delta}(s) \Delta s+\xi \mu\left(t_{2}\right) y^{\Delta}\left(t_{2}\right) \\
& \stackrel{\text { Theorem A.4 }}{=} \xi \mu\left(t_{2}\right) y^{\Delta}\left(t_{2}\right)+\int_{\sigma\left(t_{2}\right)}^{t_{3}} y^{\Delta}(s) \Delta s+(1-\eta) \mu\left(t_{3}\right) y^{\Delta}\left(t_{3}\right) \\
& +\eta \mu\left(t_{3}\right) y^{\Delta}\left(t_{3}\right)+\int_{\sigma\left(t_{3}\right)}^{T} y^{\Delta}(s) \Delta s \\
& \stackrel{(2.22),(2.30)}{\leq}\left|x\left(t^{*}\right)\right|\left[\xi \mu\left(t_{2}\right) q\left(t_{2}\right)+\int_{\sigma\left(t_{2}\right)}^{t_{3}} q(s) \Delta s+(1-\eta) \mu\left(t_{3}\right) q\left(t_{3}\right)\right. \\
& +\eta \mu\left(t_{3}\right) q\left(t_{3}\right)\left(\int_{\ell\left(t_{3}\right)}^{\sigma\left(t_{2}\right)} q(s) \Delta s-\xi \mu\left(t_{2}\right) q\left(t_{2}\right)\right) \\
& \left.+\int_{\sigma\left(t_{3}\right)}^{T} q(s)\left(\int_{\ell(s)}^{\sigma\left(t_{2}\right)} q(z) \Delta z-\xi \mu\left(t_{2}\right) q\left(t_{2}\right)\right) \Delta s\right] \\
& =\left|x\left(t^{*}\right)\right|\left[1 \cdot\left(\int_{\sigma\left(t_{2}\right)}^{\sigma\left(t_{3}\right)} q(s) \Delta s-\eta \mu\left(t_{3}\right) q\left(t_{3}\right)\right)\right. \\
& \left.+\int_{\sigma\left(t_{3}\right)}^{T} q(s)\left(\int_{\ell(s)}^{\sigma\left(t_{2}\right)} q(z) \Delta z\right) \Delta s+\eta \mu\left(t_{3}\right) q\left(t_{3}\right) \int_{\ell\left(t_{3}\right)}^{\sigma\left(t_{2}\right)} q(s) \Delta s\right] .
\end{aligned}
$$


Replace the number 1 above using (2.29) and simplify to get

$$
\begin{aligned}
y(T) \leq\left|x\left(t^{*}\right)\right|\left[\int_{\sigma\left(t_{3}\right)}^{T} q(s)\left(\int_{\ell(s)}^{\sigma\left(t_{3}\right)} q(z) \Delta z-\eta \mu\left(t_{3}\right) q\left(t_{3}\right)\right) \Delta s\right. \\
\\
\left.+\eta \mu\left(t_{3}\right) q\left(t_{3}\right)\left(\int_{\ell\left(t_{3}\right)}^{\sigma\left(t_{3}\right)} q(s) \Delta s-\eta \mu\left(t_{3}\right) q\left(t_{3}\right)\right)\right] .
\end{aligned}
$$

Use the fact that $\int_{\ell(s)}^{\sigma\left(t_{3}\right)}=\int_{\ell(s)}^{\sigma(s)}-\int_{\sigma\left(t_{3}\right)}^{\sigma(s)}$ and Lemma 2.1 to obtain

$$
\begin{aligned}
y(T) \leq\left|x\left(t^{*}\right)\right| & {\left[A\left(\int_{\sigma\left(t_{3}\right)}^{T} q(s) \Delta s+\eta \mu\left(t_{3}\right) q\left(t_{3}\right)\right)-\frac{1}{2}\left(\int_{\sigma\left(t_{3}\right)}^{T} q(s) \Delta s\right)^{2}\right.} \\
& \left.-\frac{1}{2} \int_{\sigma\left(t_{3}\right)}^{T} \mu(s) q^{2}(s) \Delta s-\eta \mu\left(t_{3}\right) q\left(t_{3}\right) \int_{\sigma\left(t_{3}\right)}^{T} q(s) \Delta s-\left(\eta \mu\left(t_{3}\right) q\left(t_{3}\right)\right)^{2}\right] \\
& \stackrel{(2.29)}{\leq}\left|x\left(t^{*}\right)\right|\left(A-\frac{1}{2}\right) \stackrel{(2.10)}{\leq}\left|x\left(t^{*}\right)\right|(1-2 \bar{p}) .
\end{aligned}
$$

Case 3. Assume that $1 / 4 \leq \bar{p} \leq 1 / 2$ and $\int_{\sigma\left(t_{2}\right)}^{T} q(s) \Delta s+\xi \mu\left(t_{2}\right) q\left(t_{2}\right) \leq B$ for $t \in \mathbb{T}$. Then, starting as in Case 1,

$$
\begin{aligned}
& y(T) \leq\left|x\left(t^{*}\right)\right|\left\{\int_{\sigma\left(t_{2}\right)}^{T} q(s)\left(\int_{\ell(s)}^{\sigma\left(t_{2}\right)} q(z) \Delta z-\xi \mu\left(t_{2}\right) q\left(t_{2}\right)\right) \Delta s\right. \\
& \left.+\xi \mu\left(t_{2}\right) q\left(t_{2}\right)\left(\int_{\ell\left(t_{2}\right)}^{\sigma\left(t_{2}\right)} q(s) \Delta s-\xi \mu\left(t_{2}\right) q\left(t_{2}\right)\right)\right\} \\
& \stackrel{(2.10)}{\leq}\left|x\left(t^{*}\right)\right|\left\{B \int_{\sigma\left(t_{2}\right)}^{T} q(s) \Delta s-\frac{1}{2}\left(\int_{\sigma\left(t_{2}\right)}^{T} q(s) \Delta s\right)^{2}-\frac{1}{2} \int_{\sigma\left(t_{2}\right)}^{T} \mu(s) q^{2}(s) \Delta s\right. \\
& \left.+\xi \mu\left(t_{2}\right) q\left(t_{2}\right)\left(B-\int_{\sigma\left(t_{2}\right)}^{T} q(s) \Delta s-\xi \mu\left(t_{2}\right) q\left(t_{2}\right)\right)\right\} \\
& \leq\left|x\left(t^{*}\right)\right|\left(B\left[\int_{\sigma\left(t_{2}\right)}^{T} q(s) \Delta s+\xi \mu\left(t_{2}\right) q\left(t_{2}\right)\right]\right. \\
& \left.-\frac{1}{2}\left[\int_{\sigma\left(t_{2}\right)}^{T} q(s) \Delta s+\xi \mu\left(t_{2}\right) q\left(t_{2}\right)\right]^{2}\right) \\
& \leq\left|x\left(t^{*}\right)\right| \frac{B^{2}}{2}=(1-2 \bar{p})\left|x\left(t^{*}\right)\right| .
\end{aligned}
$$


Neutral dynamic equations

As all three cases lead to the same contradiction, solutions $x \in \Omega$ of (1.1) must be bounded.

Theorem 2.3. Suppose there exists a constant $\bar{p} \in[0,1 / 2)$ such that $|p(t)| \leq \bar{p}$ for all $t \in \mathbb{T}$, and

$$
\int_{t_{0}}^{\infty} q(s) \Delta s=\infty
$$

If

$$
0 \leq \bar{p}<\frac{1}{4}, \quad \limsup _{t \rightarrow \infty} \int_{\ell(t)}^{\sigma(t)} q(s) \Delta s \leq \frac{3}{2}-2 \bar{p}
$$

or

$$
\frac{1}{4} \leq \bar{p}<\frac{1}{2}, \quad \limsup _{t \rightarrow \infty} \int_{\ell(t)}^{\sigma(t)} q(s) \Delta s \leq \sqrt{2(1-2 \bar{p})}
$$

then every solution $x \in \Omega$ of (1.1) goes to zero as $t \rightarrow \infty$.

Proof. Let $x \in \Omega$ be a solution of (1.1). If $x$ is nonoscillatory, assume that $x$ is eventually positive. Again select $z$ as in (2.11); then $z$ is eventually nonincreasing using (1.1). If $\bar{z}:=\lim _{t \rightarrow \infty} z(t)$, then $\bar{z}$ is bounded by Theorem 2.2 and

$$
\underset{t \rightarrow \infty}{\limsup } x(t)=\bar{z}+\limsup _{t \rightarrow \infty} p(t) x(k(t)) \leq \bar{z}+\bar{p} \limsup _{t \rightarrow \infty} x(t)
$$

so that

$$
0 \leq \limsup _{t \rightarrow \infty} x(t) \leq \frac{\bar{z}}{1-\bar{p}} \Longrightarrow \bar{z} \geq 0
$$

But from (1.1), we have

$$
\int_{t_{0}}^{\infty} q(t) x(\ell(t)) \Delta t=z\left(t_{0}\right)-\bar{z}<\infty
$$

which in view of (2.35) means that

$$
0=\liminf _{t \rightarrow \infty} x(t)=\bar{z}+\liminf _{t \rightarrow \infty}(p(t) x(k(t))) \geq \bar{z}-\bar{p} \limsup _{t \rightarrow \infty} x(t) \geq \frac{1-2 \bar{p}}{1-\bar{p}} \bar{z} \geq 0 .
$$

Thus $\bar{z}=0$ and $\lim _{t \rightarrow \infty} x(t)=0$.

If $x$ is oscillatory, by Theorem $2.2 x$ is also bounded. Set $\bar{x}:=\limsup _{t \rightarrow \infty}|x(t)|$. Then $0 \leq \bar{x}<\infty$ and $\bar{z}=\limsup _{t \rightarrow \infty}|z(t)| \geq(1-\bar{p}) \bar{x}$; without loss of generality, assume that

$$
\bar{z}:=\limsup _{t \rightarrow \infty} z(t) \geq(1-\bar{p}) \bar{x}
$$


If $\bar{x}>0$, then for any $\epsilon \in(0,(1-2 \bar{p}) \bar{x})$, there exist constants $A \in(0,3 / 2-2 \bar{p})$ and $B \in$ $(0, \sqrt{2(1-2 \bar{p})})$ and $T \in \mathbb{T}$ such that $|x(t)|<\bar{x}+\epsilon$ for $t \in\left(k^{-1}\left(\ell^{-1}(T)\right), \infty\right)_{\mathbb{T}}$ and

$$
\int_{\ell(t)}^{\sigma(t)} q(s) \Delta s \leq\left\{\begin{array}{l}
A: 0<\bar{p}<\frac{1}{4}, \\
B: \frac{1}{4} \leq \bar{p}<\frac{1}{2},
\end{array} \quad t \in[T, \infty)_{\mathbb{T}} .\right.
$$

If

$$
y(t):=z(t)-(\bar{x}+\epsilon) \bar{p} \quad \text { for } t \geq \ell(T)
$$

then

$$
-x(\ell(t))=-z(\ell(t))-p(\ell(t)) x(k(\ell(t))) \leq-z(\ell(t))+(\bar{x}+\epsilon) \bar{p}=-y(\ell(t)), \quad t \geq T .
$$

Using (1.1) and (2.44), we have

$$
y^{\Delta}(t)=z^{\Delta}(t)=-q(t) x(\ell(t)) \leq-q(t) y(\ell(t)), \quad t \geq T .
$$

Since $z^{\Delta}$ is oscillatory, $y^{\Delta}$ is too, so there exists an increasing sequence $\left\{t_{n} \in \mathbb{T}\right\}$ such that $t_{n}>k^{-1}\left(\ell^{-2}(T)\right), \lim _{n \rightarrow \infty} t_{n}=\infty, y\left(\sigma\left(t_{n}\right)\right) \rightarrow \bar{z}-(\bar{x}+\epsilon) \bar{p}>0$ as $n \rightarrow \infty$ by $(2.42)$ and (2.46), and $y^{\Delta}\left(t_{n}\right) \geq 0$. Consequently, $0 \leq y^{\Delta}\left(t_{n}\right) \leq-q\left(t_{n}\right) y\left(\ell\left(t_{n}\right)\right)$ so that

$$
y\left(\ell\left(t_{n}\right)\right) \leq 0, \quad y^{\sigma}\left(t_{n}\right)>0, \quad n \in \mathbb{N} .
$$

Hence there exists $t^{\dagger} \in\left[\ell\left(t_{n}\right), t_{n}\right]_{\mathbb{T}}$ such that either $y\left(t^{\dagger}\right)<0<y^{\sigma}\left(t^{\dagger}\right)$ or $y\left(t^{\dagger}\right)=0$. Either way, $y\left(t^{\dagger}\right) y^{\sigma}\left(t^{\dagger}\right) \leq 0$, and there exists a real number $\xi \in(0,1]$ such that

$$
y^{\sigma}\left(t^{\dagger}\right)-\xi\left[y^{\sigma}\left(t^{\dagger}\right)-y\left(t^{\dagger}\right)\right]=y\left(t^{\dagger}\right)+(1-\xi)\left[y^{\sigma}\left(t^{\dagger}\right)-y\left(t^{\dagger}\right)\right]=0 .
$$

From (2.46), we have

$$
y^{\Delta}(t) \leq-q(t) y(\ell(t)) \leq q(t)(\bar{x}+\epsilon), \quad t \in\left[T, t_{n}\right]_{\mathbb{T}},
$$

which combined with the fundamental theorem and (2.48) yields for $t \in\left[t^{\dagger}, t_{n}\right]_{\mathbb{T}}$ that

$$
\begin{aligned}
-y(\ell(t)) & =\int_{\ell(t)}^{t^{\dagger}} y^{\Delta}(s) \Delta s+(1-\xi) \mu\left(t^{\dagger}\right) y^{\Delta}\left(t^{\dagger}\right) \\
& \leq(\bar{x}+\epsilon)\left(\int_{\ell(t)}^{\sigma\left(t^{\dagger}\right)} q(s) \Delta s-\xi \mu\left(t^{\dagger}\right) q\left(t^{\dagger}\right)\right) .
\end{aligned}
$$

Put this into (2.46) to obtain

$$
y^{\Delta}(t) \leq(\bar{x}+\epsilon) q(t)\left(\int_{\ell(t)}^{\sigma\left(t^{\dagger}\right)} q(s) \Delta s-\xi \mu\left(t^{\dagger}\right) q\left(t^{\dagger}\right)\right), \quad t \in\left[t^{\dagger}, t_{n}\right]_{\mathbb{V}} .
$$


Set

$$
\lambda=\left\{\begin{array}{l}
\max \left\{A-\frac{1}{2}, \frac{1}{2}\right\}: 0 \leq \bar{p}<\frac{1}{4}, \\
\frac{B^{2}}{2}: \frac{1}{4} \leq \bar{p}<\frac{1}{2}
\end{array}\right.
$$

then $\lambda<1-2 \bar{p}$. Notice that by replacing $\left|x\left(t^{*}\right)\right|$ by $\bar{x}+\epsilon$ in the proof of Theorem 2.2, we arrive at $y\left(t_{n}\right) \leq \lambda(\bar{x}+\epsilon)$. But then by (2.44), $z\left(t_{n}\right) \leq(\bar{x}+\epsilon)(\lambda+\bar{p})$. Letting $n \rightarrow \infty$ and $\epsilon \rightarrow 0$ results in

$$
\bar{z}=\limsup _{n \rightarrow \infty} z\left(t_{n}\right) \leq(\lambda+\bar{p}) \bar{x}<(1-\bar{p}) \bar{x}
$$

a contradiction of (2.42). Therefore $\bar{x}=\limsup _{t \rightarrow \infty}|x(t)|=0$, so that any solution $x \in \Omega$ of (1.1) goes to zero.

\section{Appendix}

\section{A. Time scales}

The definitions below merely serve as a preliminary introduction to the time-scale calculus; they can be found in the context of a much more robust treatment than is allowed here in the texts $[2,3]$ and the references therein.

Definition A.1. Define the forward (backward) jump operator $\sigma(t)$ at $t$ for $t<\sup \mathbb{\mathbb { V }}$ (resp., $\rho(t)$ at $t$ for $t>\inf \mathbb{T})$ by

$$
\sigma(t)=\inf \{\tau>t: \tau \in \mathbb{T}\}, \quad(\rho(t)=\sup \{\tau<t: \tau \in \mathbb{T}\}), \quad \forall t \in \mathbb{T} .
$$

Also define $\sigma(\sup \mathbb{T})=\sup \mathbb{T}$ if $\sup \mathbb{T}<\infty$, and $\rho(\inf \mathbb{T})=\inf \mathbb{T}$ if inf $\mathbb{T}>-\infty$. Define the graininess function $\mu: \mathbb{T} \rightarrow \mathbb{R}$ by $\mu(t)=\sigma(t)-t$.

Throughout this work, the assumption is made that $\mathbb{T}$ is unbounded above and has the topology that it inherits from the standard topology on the real numbers $\mathbb{R}$. Also assume throughout that $a<b$ are points in $\mathbb{T}$ and define the time scale interval $[a, b]_{\mathbb{T}}=\{t \in$ $\mathbb{T}: a \leq t \leq b\}$. The jump operators $\sigma$ and $\rho$ allow the classification of points in a time scale in the following way. If $\sigma(t)>t$, the point $t$ is right-scattered, while if $\rho(t)<t$ then $t$ is left-scattered. If $\sigma(t)=t$, the point $t$ is right-dense; if $t>\inf \mathbb{T}$ and $\rho(t)=t$, then $t$ is left-dense.

Definition A.2. Fix $t \in \mathbb{T}$ and let $y: \mathbb{T} \rightarrow \mathbb{R}$. Define $y^{\Delta}(t)$ to be the number (if it exists) with the property that given that $\epsilon>0$, there is a neighbourhood $U$ of $t$ such that for all $s \in U$,

$$
\left|[y(\sigma(t))-y(s)]-y^{\Delta}(t)[\sigma(t)-s]\right| \leq \epsilon|\sigma(t)-s|
$$

Call $y^{\Delta}(t)$ the (delta) derivative of $y$ at $t$. 
Definition A.3. If $F^{\Delta}(t)=f(t)$, then define the (Cauchy) delta integral by

$$
\int_{a}^{t} f(s) \Delta s=F(t)-F(a)
$$

The following theorem is due to Hilger [6].

Theorem A.4. Assume that $f: \mathbb{T} \rightarrow \mathbb{R}$ and let $t \in \mathbb{T}$.

(1) If $f$ is differentiable at then $f$ is continuous at $t$.

(2) If $f$ is continuous at $t$ and $t$ is right-scattered, then $f$ is differentiable at $t$ with

$$
f^{\Delta}(t)=\frac{f(\sigma(t))-f(t)}{\sigma(t)-t}
$$

(3) If $f$ is differentiable and $t$ is right-dense, then

$$
f^{\Delta}(t)=\lim _{s \rightarrow t} \frac{f(t)-f(s)}{t-s} .
$$

(4) If $f$ is differentiable at $t$, then $f(\sigma(t))=f(t)+\mu(t) f^{\Delta}(t)$.

Definition A.5. A function $f: \mathbb{T} \rightarrow \mathbb{R}$ is right-dense continuous (denoted by $f \in C_{r d}(\mathbb{T} ; \mathbb{R})$ ) provided that $f$ is continuous at every right-dense point $t \in \mathbb{T}$, and $\lim _{s \rightarrow t^{-}} f(s)$ exists and is finite at every left-dense point $t \in \mathbb{T}$.

According to [2, Theorem 1.74], every right-dense continuous function has a delta antiderivative. This implies that the delta definite integral of any right-dense continuous function exists.

\section{References}

[1] D. R. Anderson, R. J. Krueger, and A. C. Peterson, Delay dynamic equations with stability, Advances in Difference Equations 2006 (2006), Article ID 94051, 19 pages.

[2] L. Berezansky and E. Braverman, Oscillation of a logistic difference equation with several delays, Advances in Difference Equations 2006 (2006), Article ID 82143, 12 pages.

[3] M. Bohner and A. C. Peterson, Dynamic Equations on Time Scales, An Introduction with Applications, Birkhäuser, Massachusetts, 2001.

[4] M. Bohner and A. C. Peterson (eds.), Advances in Dynamic Equations on Time Scales, Birkhäuser, Massachusetts, 2003.

[5] L. H. Erbe, H. Xia, and J. S. Yu, Global stability of a linear nonautonomous delay difference equation, Journal of Difference Equations and Applications 1 (1995), no. 2, 151-161.

[6] S. Hilger, Analysis on measure chains - a unified approach to continuous and discrete calculus, Results in Mathematics 18 (1990), no. 1-2, 18-56.

Douglas R. Anderson: Department of Mathematics and Computer Science, Concordia College,

Moorhead, MN 56562, USA

E-mail address: andersod@cord.edu 